\title{
SOME STRANGE FEATURES OF THE GALILEI GROUP
}

\author{
BARBARA GOŁUBOWSKA, VASYL KOVALCHUK, AGNIESZKA \\ MARTENS, EWA ELIZA ROŻKO AND JAN J. SŁAWIANOWSKI
}

Presented by Jan J. Sławianowski

\begin{abstract}
Discussed are certain strange properties of the Galilei group, connected first of all with the property of mechanical energy-momentum covector to be an affine object, rather than the linear one. Its affine transformation rule is interesting in itself and dependent on the particle mass. On the quantum level this means obviously that we deal with the projective unitary representation of the group rather than with the usual representation. The status of mass is completely different than in relativistic theory, where it is a continuous eigenvalue of the Casimir invariant. In Galilei framework it is a parameter characterizing the factor of the projective representation, in the sense of V. Bargmann. This "pathology" from the relativistic point of view is nevertheless very interesting and it underlies the Weyl-WignerMoyal-Ville approach to quantum mechanics.
\end{abstract}

\section{Introduction to the Galilei Group}

It was shown that when some natural postulates are accepted, then there are only two natural flat space-time structures: Galilean and Minkowskian. And, respectively, there are two alternative symmetry groups: Galilei and Poincare groups. The peculiarity of Minkowski-Poincare structure is the existence of some fixed universal physical constant-velocity of light $c$. In a sense the traditional Galilei structure is obtained in the limit transition $c \rightarrow \infty$. And in fact there are numerous formulae in which this limit is smoothly achieved. But as usual when it is a "true" limit transition to some singular value, certain discontinuities in the limit appear and the resulting theory changes drastically. The asymptotic form of the theory is not its merely special case and important qualitative discontinuities appear. The same, and even more catastrophically, happens in the $\hbar \rightarrow 0$ asymptotics of quantum mechanics. Classical mechanics asymptotically corresponds to quantum-mechanical results, but nevertheless it is a qualitatively different theory. In the space-time theory the main peculiarity of the limit transition $c \rightarrow \infty$ is the catastrophic change of the structure of the four-momentum obtained on the basis of the four-dimensional Legendre transformation. In this sense the relativistic theory 
is simpler, its four-momentum is the usual covector. In Galilean framework, it becomes an affine geometric object rather than linear one, if its transformation rule is to be compatible with its physical interpretation of energy-momentum. From this point of view the linear covector rule is drastically false.

Let us begin with some formalism. Galilean space is an affine manifold endowed with some additional structures: $(X, V, \rightarrow, S, g, \delta)$. Here $X$ denotes the point set of the manifold, $V$ is the linear space of translations in $X, \rightarrow$ is the operation of the vector between a pair of points, $S \subset V$ is a linear subspace of co-dimension one, and $g \in S^{*} \otimes S^{*}, \delta \in(V / S)^{*} \otimes(V / S)^{*}$ are twice covariant symmetric tensors in $S$ and $V / S$. As always in affine geometry the operation $\rightarrow: X \times X \rightarrow V$ is assumed to satisfy the following axioms

$$
\overrightarrow{x y}+\overrightarrow{y z}+\overrightarrow{z x}=0 \quad \text { for any } \quad x, y, z \in X
$$

and for any $x \in X$ the mapping

$$
X \ni y \mapsto \overrightarrow{x y} \in V
$$

is a bijection of $X$ onto $V$. In affine spaces over the field of reals $\mathbb{R}$ this implies that

$$
\overrightarrow{x y}=-\vec{y} \vec{x}, \quad \vec{x} \vec{x}=0
$$

for any $x, y \in X$. It is important that the metric $g$ is defined merely on $S$. The pairs of points translated by elements of $S$ are said to be simultaneous and it is important that the metric tensor $g$ defines the distances and scalar products only for simultaneous pairs of points and for vectors in $S$. There is no concept of distance between non-simultaneous events. Physically $X$ is four-dimensional and $S$ is three-dimensional, nevertheless in many general statements this does not matter. Obviously, the operation $\rightarrow$ establishes the action of $V$ as an Abelian additive transformation group on $X$. Those translations, $t[v]: X \rightarrow X$ act as follows

$$
t[\overrightarrow{x y}](x)=y
$$

for any $x, y \in X$. Therefore, being a subgroup $S \subset V$, the space $S$ acts nontransitively as the transformation group, and the quotient

$$
T:=X / t[S]
$$

is a one-dimensional affine space with the naturally induced affine structure. The natural projection of $X$ onto $T$ and the induced one of $V$ onto $V / S$ will be denoted by

$$
\Pi: X \rightarrow T, \quad \pi: V \rightarrow V / S .
$$


The one-dimensional linear space of translations in $T$ is canonically identical with $V / S$, if necessary we shall denote it by

$$
Z=V / S
$$

Let us stress that the Galilei space-time is a fibre structure over the time axis $T$, however, unlike the structure called sometimes the Aristotle space-time it is not a Cartesian product of time and space. There are "spatial", i.e., "along $\mathrm{t}[S]$ "directions, but there is no well-defined concept of position, so there is no space. And spatial distances are defined only for simultaneous, i.e., $t[S]$-related events

$$
\mathrm{d}_{S}(x, y)=\sqrt{g(\overrightarrow{x y}, \overrightarrow{x y})}=\sqrt{g_{i j}\left(y^{i}-x^{i}\right)\left(y^{j}-x^{j}\right)} \quad \text { if } \quad \Pi(x)=\Pi(y) .
$$

And similarly, the absolute time distance of two arbitrary events is

$$
\mathrm{d}_{T}(x y)=\sqrt{\delta(\pi(\overrightarrow{x y}), \pi(\overrightarrow{x y}))} .
$$

This structure is simultaneously too weak and too strong, depending on analyzed problems. Too weak because no orientation is fixed, in particular no spatial orientation and no arrow of time. If necessary, they should be defined, fixed in $T, S$ or just in $X$. But at the same time it is too strong, namely, when fixing spatial metric. Let us remind the ideas by Hehl, Ne'emann, Sijacki and our own ones to formulate the affinely-invariant mechanics and physics. Those ideas are still compatible with the main fibre-bundle structure of the Galilei space. Namely, just removing the objects $g, \delta$, i.e., metric tensors, we obtain the amorphous, affine-Galilei structure. This description was more or less complete. We presented it in details just to show you that unlike the everyday opinions the non-relativistic Galilean space-time is geometrically incomparatively more complicated than the relativistic PoincareMinkowski space-time. But if motion is relative, and if, as we know from experiment, the inertial, i.e., uniform rectilinear motion is physically indistinguishable from the rest state and depends only on the choice of the reference frame, then really it must be so. Let us now fix the reference frame and present the analytical description. When the origin of the reference frame and some rest-state standard are fixed, and so are physical units, then $X$ becomes identified with the Cartesian product of time and space, e.g.

$$
X=\mathbb{R} \times S
$$

In other words, the space-time events are represented by the Aristotle-Newton pairs: instancy of the time-position vector. There is nothing bad in this identification if we remember it is merely something like the choice of coordinates. 
Galilei transformations are defined as the automorphisms of the Galilean spacetime. In the mentioned identification they are given by

$$
\left[\begin{array}{c}
t \\
\bar{x}
\end{array}\right] \mapsto\left[\begin{array}{c}
t+\varepsilon \\
R \bar{x}+\bar{\nu} t+\bar{\alpha}
\end{array}\right]
$$

Here $[\varepsilon, \bar{\alpha}]^{T}$ is the space-time translation vector, $\bar{\nu}$ is the spatial vector of boost, and $R \in \mathrm{O}(n-1, \mathbb{R})$ is the spatial rotation. So, analytically we write (11) as

$$
\left[\begin{array}{c}
t \\
x^{i}
\end{array}\right] \mapsto\left[\begin{array}{c}
t+\varepsilon \\
R_{j}^{i} x^{j}+\nu^{i} t+\alpha^{i}
\end{array}\right] .
$$

Here the summation convention is meant under spatial indices $j=\overline{1,(n-1)}$. The time dimension is in analogy to relativity denoted as zeroth or simply by the index $t$. The matrix $R$ is orthogonal, so

$$
\delta_{i j} R_{k}^{i} R_{l}^{j}=\delta_{k l}
$$

or less correctly $R^{T} R=I$. As mentioned, in physics $n=4$, nevertheless it is more convenient to write certain formulae for the "general" $n$.

The Galilei transformation (11), (12) will be denoted by $\mathcal{G}[R, \bar{\nu} ; \varepsilon, \bar{\alpha}]$. The structure of the Galilei group is rather complicated and even its homogeneous part fails to be semisimple. Let us denote this group shortly by $\operatorname{Gal}(X ; g, \delta)$ or just by $\operatorname{Gal}(X)$ when there is no danger of misunderstanding.

One can show that the composition rule for the Galilei transformations (11), (12) may be written down as follows

$$
\mathcal{G}\left[R_{1}, \bar{\nu}_{1} ; \varepsilon_{1}, \bar{\alpha}_{1}\right] \mathcal{G}\left[R_{2}, \bar{\nu}_{2} ; \varepsilon_{2}, \bar{\alpha}_{2}\right]=\mathcal{G}[R, \bar{\nu} ; \varepsilon, \bar{\alpha}] .
$$

Here

$$
R=R_{1} R_{2}, \quad \bar{\nu}=\bar{\nu}_{1}+R \bar{\nu}_{2}, \quad \varepsilon=\varepsilon_{1}+\varepsilon_{2}, \quad \bar{\alpha}=\bar{\alpha}_{1}+R_{1} \bar{\alpha}_{2}+\bar{\nu}_{1} \varepsilon_{2} .
$$

The inverse mapping is given by

$$
\mathcal{G}[R, \bar{\nu} ; \varepsilon, \bar{\alpha}]^{-1}=\mathcal{G}[\widetilde{R}, \widetilde{\bar{\nu}} ; \widetilde{\varepsilon}, \widetilde{\bar{\alpha}}] .
$$

Here

$$
\widetilde{R}=R^{-1}, \quad \widetilde{\bar{\nu}}=-R^{-1} \bar{\nu}, \quad \widetilde{\varepsilon}=-\varepsilon, \quad \widetilde{\bar{\alpha}}=-R^{-1}(\bar{\alpha}-\bar{\nu} \varepsilon) .
$$

We see that in fact the composition rule is rather complicated and contains a few floors of semi-direct rules even in the homogeneous part. 
Obviously, in $n$-dimensional Galilean space we have

$$
\operatorname{dim} \operatorname{Gal}(X)=\frac{n(n+1)}{2}, \quad \operatorname{dim} \mathrm{O}(n, \mathbb{R})=\frac{(n-1)(n-2)}{2} .
$$

Therefore in four dimensions the Galilei group is 10-dimensional and the group of spatial rotations is three-dimensional.

It is clear that Galilean transformations (11) acting on the straight-line of the uniform rectilinear motion with velocity $\bar{v}$, transform it into one with the new velocity

$$
\bar{v}^{\prime}=R \bar{v}+\bar{\nu} .
$$

If $R=\operatorname{Id}_{n}$, this is simply the Galilean boost

$$
\bar{v}^{\prime}=\bar{v}+\bar{\nu} .
$$

As mentioned, Galilean space-time is not the Cartesian product of time and space, our description above is a merely parametrization. Because of this the threedimensional velocity is not a true three-dimensional vector. The space of velocities is not linear, it is an affine space. But the fact that two uniform rectilinear motions differ by $\bar{\nu}$ in velocity is objective. It has to do with that the $(n-1)$-dimensional (physically three-dimensional) group of boosts is a normal subgroup of the full Galilei group.

Let us make now a digression concerning the amorphous Galilei group. It consists of transformations $G[\lambda, A, \bar{\nu} ; \varepsilon, \bar{\alpha}]$ of $\operatorname{Gal}(X)$ given by

$$
G[\lambda, A, \bar{\nu} ; \varepsilon, \bar{\alpha}]\left[\begin{array}{c}
t \\
\bar{x}
\end{array}\right]=\left[\begin{array}{c}
\lambda t+\varepsilon \\
A \bar{x}+\bar{\nu} t+\bar{\alpha}
\end{array}\right] .
$$

Here $\lambda \in \mathbb{R} \backslash\{0\}, A \in \mathrm{GL}(n-1, \mathbb{R})$, and $\varepsilon, \bar{\nu}, \bar{\alpha}$ have the same meaning as in the usual, i.e., "metrical", Galilei group. Here $\lambda$ is the temporal dilatational factor and $A$ is a spatial linear transformation. And again the problem appears of admitting the reversal of time, $\lambda<0$ and the change of spatial orientation, $\operatorname{det} A<0$.

The general structure of the amorphous Galilei group is similar to that of the "metrical" group. The complicated hierarchy of semi-direct products is just analogous to what were seeing there.

\section{Analytical Description and Infinitesimal Rules}

To understand correctly what we have called "the strange features of the Galilei group", its infinitesimal transformations and the difference between it and Poincare 
group, it is convenient to use the matrix representation of the group elements and of the basic generators of the Lie algebra. To take inhomogeneous transformations into account, it is convenient to use $(n+1) \times(n+1)$ matricesi.e., in the physically interesting case one has $5 \times 5$ matrix.

Obviously, the linear representation of affine Galilean mappings

$$
\left[\begin{array}{ccc}
1 & \overline{\mathcal{O}}^{T} & \varepsilon \\
\bar{\nu} & R & \bar{\alpha} \\
0 & \overline{\mathcal{O}}^{T} & 1
\end{array}\right], \quad\left[\begin{array}{ccc}
\lambda & \overline{\mathcal{O}}^{T} & \varepsilon \\
\bar{\nu} & L & \bar{\alpha} \\
0 & \overline{\mathcal{O}}^{T} & 1
\end{array}\right]
$$

respectively for the metrical and amorphous groups. Here $\bar{\nu}, \bar{\alpha}, \overline{\mathcal{O}}$ denote the column matrices; $R$ and $L$ denote respectively the $(n-1) \times(n-1)$ orthogonal and general non-singular matrices.

In four dimensions infinitesimal generators of the homogeneous transformations are given respectively by

$$
M_{1}=\left[\begin{array}{cccc}
0 & 0 & 0 & 0 \\
0 & 0 & 0 & 0 \\
0 & 0 & 0 & -1 \\
0 & 0 & 1 & 0
\end{array}\right], \quad M_{2}=\left[\begin{array}{cccc}
0 & 0 & 0 & 0 \\
0 & 0 & 0 & 1 \\
0 & 0 & 0 & 0 \\
0 & -1 & 0 & 0
\end{array}\right], \quad M_{3}=\left[\begin{array}{cccc}
0 & 0 & 0 & 0 \\
0 & 0 & -1 & 0 \\
0 & 1 & 0 & 0 \\
0 & 0 & 0 & 0
\end{array}\right]
$$

for rotations, and

$$
N_{1}=\left[\begin{array}{llll}
0 & 0 & 0 & 0 \\
1 & 0 & 0 & 0 \\
0 & 0 & 0 & 0 \\
0 & 0 & 0 & 0
\end{array}\right], \quad N_{2}=\left[\begin{array}{llll}
0 & 0 & 0 & 0 \\
0 & 0 & 0 & 0 \\
1 & 0 & 0 & 0 \\
0 & 0 & 0 & 0
\end{array}\right], \quad N_{3}=\left[\begin{array}{llll}
0 & 0 & 0 & 0 \\
0 & 0 & 0 & 0 \\
0 & 0 & 0 & 0 \\
1 & 0 & 0 & 0
\end{array}\right]
$$

for boosts.

Obviously, $M_{a}$ generate rotations about the $a$-th axis, i.e., in the $(b, c)$-plane where $a \neq b \neq c \neq a$. So, in dimensions of space-time higher than four one should have used rather the symbol $M_{b c}=-M_{c b}$ for them. The matrices $N_{a}$ generate boots along the $a$-th axis. The commutation rules are given by

$$
\left[M_{a}, M_{b}\right]=\varepsilon_{a b}^{c} M_{c}, \quad\left[M_{a}, N_{b}\right]=\varepsilon_{a b}^{c} N_{c}, \quad\left[N_{a}, N_{b}\right]=0 .
$$

Let us stress that unlike the situation one is faced with in relativistic theory, this Lie algebra is not semisimple. It is isomorphic with the Lie algebra of Euclidean group in $(n-1)$ dimensions where the boots are playing the role of translations. For the one-parameter subgroups generated by $M_{a}, N_{a}$

$$
\exp \left(\varphi n^{a} M_{a}\right), \quad \exp \left(\nu n^{a} N_{a}\right), \quad \bar{n} \cdot \bar{n}=1
$$


the first-kind canonical parameters $\varphi, \nu$ are playing respectively the role of the angle of rotation and velocity.

In the four space-time dimensions the $5 \times 5$ matrices of the Galilean generators are given by the following ten matrices

$$
\begin{aligned}
& \mathbb{M}_{1}=\left[\begin{array}{ccccc}
0 & 0 & 0 & 0 & 0 \\
0 & 0 & 0 & 0 & 0 \\
0 & 0 & 0 & -1 & 0 \\
0 & 0 & 1 & 0 & 0 \\
0 & 0 & 0 & 0 & 0
\end{array}\right], \quad \mathbb{M}_{2}=\left[\begin{array}{ccccc}
0 & 0 & 0 & 0 & 0 \\
0 & 0 & 0 & 1 & 0 \\
0 & 0 & 0 & 0 & 0 \\
0 & -1 & 0 & 0 & 0 \\
0 & 0 & 0 & 0 & 0
\end{array}\right] \\
& \mathbb{M}_{3}=\left[\begin{array}{ccccc}
0 & 0 & 0 & 0 & 0 \\
0 & 0 & -1 & 0 & 0 \\
0 & 1 & 0 & 0 & 0 \\
0 & 0 & 0 & 0 & 0 \\
0 & 0 & 0 & 0 & 0
\end{array}\right], \quad \mathbb{N}_{1}=\left[\begin{array}{lllll}
0 & 0 & 0 & 0 & 0 \\
1 & 0 & 0 & 0 & 0 \\
0 & 0 & 0 & 0 & 0 \\
0 & 0 & 0 & 0 & 0 \\
0 & 0 & 0 & 0 & 0
\end{array}\right] \\
& \mathbb{N}_{2}=\left[\begin{array}{lllll}
0 & 0 & 0 & 0 & 0 \\
0 & 0 & 0 & 0 & 0 \\
1 & 0 & 0 & 0 & 0 \\
0 & 0 & 0 & 0 & 0 \\
0 & 0 & 0 & 0 & 0
\end{array}\right], \quad \mathbb{N}_{3}=\left[\begin{array}{lllll}
0 & 0 & 0 & 0 & 0 \\
0 & 0 & 0 & 0 & 0 \\
0 & 0 & 0 & 0 & 0 \\
1 & 0 & 0 & 0 & 0 \\
0 & 0 & 0 & 0 & 0
\end{array}\right] \\
& \mathbb{P}_{1}=\left[\begin{array}{lllll}
0 & 0 & 0 & 0 & 0 \\
0 & 0 & 0 & 0 & 1 \\
0 & 0 & 0 & 0 & 0 \\
0 & 0 & 0 & 0 & 0 \\
0 & 0 & 0 & 0 & 0
\end{array}\right], \quad \mathbb{P}_{2}=\left[\begin{array}{lllll}
0 & 0 & 0 & 0 & 0 \\
0 & 0 & 0 & 0 & 0 \\
0 & 0 & 0 & 0 & 1 \\
0 & 0 & 0 & 0 & 0 \\
0 & 0 & 0 & 0 & 0
\end{array}\right] \\
& \mathbb{P}_{3}=\left[\begin{array}{lllll}
0 & 0 & 0 & 0 & 0 \\
0 & 0 & 0 & 0 & 0 \\
0 & 0 & 0 & 0 & 0 \\
0 & 0 & 0 & 0 & 1 \\
0 & 0 & 0 & 0 & 0
\end{array}\right], \quad \mathbb{P}_{t}=\left[\begin{array}{lllll}
0 & 0 & 0 & 0 & 1 \\
0 & 0 & 0 & 0 & 0 \\
0 & 0 & 0 & 0 & 0 \\
0 & 0 & 0 & 0 & 0 \\
0 & 0 & 0 & 0 & 0
\end{array}\right]=\mathbb{T}
\end{aligned}
$$

Their commutation relations have the form

$$
\begin{aligned}
& {\left[\mathbb{M}_{a}, \mathbb{M}_{b}\right]=\varepsilon_{a b}{ }^{c} \mathbb{M}_{c}, \quad\left[\mathbb{M}_{a}, \mathbb{N}_{b}\right]=\varepsilon_{a b}{ }^{c} \mathbb{N}_{c}, \quad\left[\mathbb{M}_{a}, \mathbb{P}_{b}\right]=\varepsilon_{a b}{ }^{c} \mathbb{P}_{c}} \\
& {\left[\mathbb{N}_{a}, \mathbb{N}_{b}\right]=0, \quad\left[\mathbb{N}_{a}, \mathbb{P}_{b}\right]=0, \quad\left[\mathbb{P}_{a}, \mathbb{P}_{b}\right]=0} \\
& {\left[\mathbb{M}_{a}, \mathbb{T}\right]=0, \quad\left[\mathbb{N}_{a}, \mathbb{T}\right]=\mathbb{P}_{a}, \quad\left[\mathbb{P}_{a}, \mathbb{T}\right]=0 .}
\end{aligned}
$$

Later on, when looking at similar expressions for the Poincare group, we shall conclude how the "small" modification of Lie-algebraic rules changes catastrophically the group structure. 
Let us review in addition certain important finite commutation rules for the Galilei group, more precisely those concerning its normal subgroups:

$$
\begin{aligned}
\mathcal{G}[R, \bar{\nu} ; \varepsilon, \bar{\alpha}] \mathcal{G}[I, \overline{\mathcal{O}} ; \tau, \bar{\xi}] \mathcal{G}[R, \bar{\nu} ; \varepsilon, \bar{\alpha}]^{-1} & =\mathcal{G}[I, \overline{\mathcal{O}} ; \tau, R \bar{\xi}+\tau \bar{\nu}] \\
\mathcal{G}[R, \bar{\nu} ; \varepsilon, \bar{\alpha}] \mathcal{G}[I, \overline{\mathcal{O}} ; 0, \bar{\xi}] \mathcal{G}[R, \bar{\nu} ; \varepsilon, \bar{\alpha}]^{-1} & =\mathcal{G}[I, \overline{\mathcal{O}} ; 0, R \bar{\xi}] \\
\mathcal{G}[R, \bar{\nu} ; \varepsilon, \bar{\alpha}] \mathcal{G}[I, \overline{\mathcal{O}} ; \tau, \overline{\mathcal{O}}] \mathcal{G}[R, \bar{\nu} ; \varepsilon, \bar{\alpha}]^{-1} & =\mathcal{G}[I, \overline{\mathcal{O}} ; \tau, \tau \bar{\nu}] \\
\mathcal{G}[R, \bar{\nu} ; \varepsilon, \bar{\alpha}] \mathcal{G}[I, \bar{\mu} ; 0, \bar{\xi}] \mathcal{G}[R, \bar{\nu} ; \varepsilon, \bar{\alpha}]^{-1} & =\mathcal{G}[I, R \bar{\mu} ; 0, R \bar{\xi}] \\
\mathcal{G}[R, \bar{\nu} ; \varepsilon, \bar{\alpha}] \mathcal{G}[I, \bar{\mu} ; \tau, \bar{\xi}] \mathcal{G}[R, \bar{\nu} ; \varepsilon, \bar{\alpha}]^{-1} & =\mathcal{G}[I, R \bar{\mu} ; \tau, R \bar{\xi}+\tau \bar{\nu}-\varepsilon R \bar{\mu}] \\
\mathcal{G}[R, \bar{\nu} ; \varepsilon, \bar{\alpha}] \mathcal{G}[I, \bar{\mu} ; 0, \overline{\mathcal{O}}] \mathcal{G}[R, \bar{\nu} ; \varepsilon, \bar{\alpha}]^{-1} & =\mathcal{G}[I, R \bar{\mu} ; 0, \overline{\mathcal{O}}]
\end{aligned}
$$

Let us comment briefly those rules. The equation (35) tells us that the space-time translations form a normal subgroup. Moreover, it follows from (36) that also the group of spatial translations is normal. But unlike this, (37) means that the time translations fail to be normal group. And this was expected: spatial translations, i.e., $t[S]$-translations are objectively defined, whereas the time translations need defining a reference frame to be defined in Galilei space. An important feature is (38). It tells us that the Newton translations, i.e., ones in positions and velocities form a normal subgroup. This fact is very important physically. The Abelian group of newton translations acts freely and transitively in the $2(n-1)$-dimensional phase space of initial conditions. Let us mention that among other features, this is essential for the formulating the phase-space approach to quantum mechanics. Let us mention also that being $2(n-1)$-dimensional it is too large to admit a true Hamiltonian representation. On the quantum level this means that the theory is not based on the unitary representations, but on the projective (ray) representation of the Newton group. The mass parameter does not occur as a Casimir invariant, as it does in relativistic theory, but as a parameter which labels the projective rule of composition. (39) means that the $(2 n-1)$-dimensional group of Newton translations and time translations is a normal subgroup. And from (40) it follows that so is the Abelian group of boosts.

Let us consider a mechanical system with $f$ degrees of freedom and generalized coordinates $q^{1}, \ldots, q^{f}$. Its dynamics is encoded in the Lagrangian $L\left(t, q^{i}, \frac{\mathrm{d} q^{i}}{\mathrm{~d} t}\right)$, where $q^{i}, \mathrm{~d} q^{i} / \mathrm{d} t$ is an abbreviation for the dependence on all coordinates and generalized velocities. Obviously, the variable $t$ denotes the absolute time. The action functional for problems with fixed boundary condition is given by

$$
I[q]=\int_{t_{\text {in }}}^{t_{\text {fin }}} L\left(t, q^{i}, \frac{\mathrm{d} q^{i}}{\mathrm{~d} t}\right) \mathrm{d} t
$$


and the resulting equations of motion have the form

$$
\frac{\delta I}{\delta q^{i}(t)}=\frac{\partial L}{\partial q^{i}}-\frac{D}{D t} \frac{\partial L}{\partial \dot{q}^{i}}=0 .
$$

Now let us go to the homogeneous formalism [11,12]. It is interesting in itself, it enables one to go smoothly to the relativistic theory, and it simply very convenient if not necessary when discussing time dependent dynamical problems. And it is just fundamental for our understanding of the problems with Galilei group. So, let us include the time variable $t$, together with $q^{i}$, on equal footing into space-time coordinates. To describe motion we introduce a new independent variable $\tau-$ an arbitrary parameter, in general quite "non-physical" one. Let us transform our variational principle to this new description

$$
\begin{aligned}
\int L\left(t, q^{i}(t), \frac{\mathrm{d} q^{i}}{\mathrm{~d} t}(t)\right) \mathrm{d} t & =\int L\left(t(\tau), q^{i}(\tau), \frac{\mathrm{d} q^{i}}{\mathrm{~d} \tau}(\tau)\left[\frac{\mathrm{d} t}{\mathrm{~d} \tau}\right]^{-1}\right) \mathrm{d} \tau \\
& =\int \Lambda\left(t(\tau), q^{i}(\tau), \frac{\mathrm{d} t}{\mathrm{~d} \tau}(\tau), \frac{\mathrm{d} q^{i}}{\mathrm{~d} \tau}(\tau)\right) \mathrm{d} \tau
\end{aligned}
$$

Let us now introduce the parametric "generalized velocities":

$$
u^{t}=\frac{\mathrm{d} t}{\mathrm{~d} \tau}, \quad u^{i}=\frac{\mathrm{d} q^{i}}{\mathrm{~d} t} \frac{\mathrm{d} t}{\mathrm{~d} \tau}=v^{i} u^{t}, \quad v^{i}=\frac{u^{i}}{u^{t}}, \quad i=1, \ldots, f .
$$

Therefore

$$
\Lambda\left(t, q^{i}, u^{t}, u^{i}\right)=L\left(t(\tau), q^{i}(\tau), \frac{u^{i}(\tau)}{u^{t}(\tau)}\right) u^{t}(\tau) .
$$

This is the new homogeneous Lagrangian. $\Lambda$ is really homogeneous of degree one in " $\tau$-parametric velocities":

$$
\Lambda\left(t, q^{i}, a u^{t}, a u^{i}\right)=a \Lambda\left(t, q^{i}, u^{t}, u^{i}\right), \quad a>0 .
$$

From now on we can forget about its origin and just to reconstruct the whole system of analytical mechanics. First of all we introduce a homogeneous Legendre transformation:

$$
\mathcal{L}: \quad p_{t}=\frac{\partial \Lambda}{\partial u^{t}}, \quad p_{i}=\frac{\partial \Lambda}{\partial u^{i}}, \quad i=1, \ldots, f .
$$

This transformation is homogeneous of degree zero in $\tau$-velocities.

Let us only mention that there is some historical ambiguity, namely how to refer to $u^{t}: u^{0}$ or $u^{f+1}$ ? But it is not very essential for us. 
Then one can show that

$$
\begin{aligned}
& p_{t}=L\left(t, q^{i}, v^{i}\right)-v^{i} \frac{\partial L}{\partial v^{i}}\left(t, q^{j}, v^{j}\right)=-E \\
& p_{i}=\frac{\partial L}{\partial v^{i}}\left(t, q^{k}, v^{k}\right) .
\end{aligned}
$$

Therefore

$$
p_{t} \mathrm{~d} t+p_{i} \mathrm{~d} q^{i}=-E \mathrm{~d} t+p_{i} \mathrm{~d} q^{i} .
$$

Here $E$ is physically interpreted as energy and we have the following conjugation scheme

$$
\left(-E, p_{i}\right) \sim\left(t, q^{i}\right) .
$$

In the $(2 f+2)$-dimensional QTPH-space in the sense of Synge $[5,11,12]$ parametrized by coordinates $\left(t, q^{i}, p_{t}, p_{i}\right)$ as canonical variables the above Legendre transformation induces the constraints of dimension $(2 f+1)$ given by the following equations

$$
\Omega=p_{t}+H\left(t, q^{i}, p_{i}\right)=0 .
$$

Here

$$
H\left(t, q^{i}, p_{i}\right)=E\left(t, q^{i}, p_{i}\right)
$$

and

$$
p_{i}=\frac{\partial L}{\partial v^{i}}, \quad q^{i}=\frac{\partial H}{\partial p_{i}} .
$$

Hamiltonian equations of motion reduce then to the following well-known systems

$$
\begin{aligned}
\frac{\mathrm{d} q^{i}}{\mathrm{~d} \tau}=\frac{\partial \Omega}{\partial p_{i}}=\frac{\partial H}{\partial p_{i}}, & \frac{\mathrm{d} p_{i}}{\mathrm{~d} \tau}=-\frac{\partial \Omega}{\partial q^{i}}=-\frac{\partial H}{\partial q^{i}} \\
\frac{\mathrm{d} t}{\mathrm{~d} \tau}=\frac{\partial \Omega}{\partial p_{\tau}}=1, & \frac{\mathrm{d} p_{t}}{\mathrm{~d} \tau}=-\frac{\partial H}{\partial t} .
\end{aligned}
$$

But it is interesting to investigate the relationship between the usual and homogeneous Lagrange formalism.

Let us write down the system of homogeneous Lagrange equations

$$
\frac{D}{D \tau} \frac{\partial \Lambda}{\partial u^{t}}-\frac{\partial \Lambda}{\partial t}=0, \quad \frac{D}{D \tau} \frac{\partial \Lambda}{\partial u^{i}}-\frac{\partial \Lambda}{\partial q^{i}}=0 .
$$

This is evidently a dependent system. The first subsystem is simply equivalent to

$$
\frac{D E}{D t}=-\frac{\partial L}{\partial t}
$$


This is simply the energy conservation law. The second subsystem of (57) implies that

$$
\frac{D}{D t} \frac{\partial L}{\partial v^{i}}-\frac{\partial L}{\partial q^{i}}=-\frac{\mathrm{d} t}{\mathrm{~d} \tau} \frac{\mathrm{d}^{2} \tau}{\mathrm{d} t^{2}} \frac{\partial L}{\partial v^{i}} .
$$

It is clear that if $t=\tau$, or at least when $\tau$ is linear in $t$ ( $t$ is linear in $\tau$ ), then (59) reduces to the usual system of Euler-Lagrange equations

$$
\frac{D}{D t} \frac{\partial L}{\partial v^{i}}-\frac{\partial L}{\partial q^{i}}=0
$$

\section{Elements of the Relativistic Description, Momentum Four-Vector}

Now, for comparison let us consider the relativistic homogeneous formalism. Traditional Lagrangian looks a bit artificial

$$
L=-m c^{2} \sqrt{1-\frac{v^{2}}{c^{2}}}=-m c^{2} \sqrt{1-\stackrel{(3)}{g}_{i j} \frac{\mathrm{d} x^{i}}{\mathrm{~d} t} \frac{\mathrm{d} x^{j}}{\mathrm{~d} t}} .
$$

Here $\stackrel{(3)}{g}$ is the three-dimensional part of the four-dimensional Minkowskian metric $\left[g_{\mu \nu}\right]$.

The corresponding homogeneous Lagrangian, the only physically justified in this kind of problems, is given by

$$
\Lambda=-m c \sqrt{g_{\mu \nu} \frac{\mathrm{d} x^{\mu}}{\mathrm{d} \theta} \frac{\mathrm{d} x^{\nu}}{\mathrm{d} \theta}} .
$$

Therefore, the action functional is given by

$$
\mathrm{I}=\int \Lambda \mathrm{d} \theta
$$

Here

$$
\Lambda=-m c \sqrt{g_{\mu \nu} u^{\mu} u^{\nu}}
$$

and $\theta$ is an arbitrary parameter.

Let us now perform the formal Legendre transformation

$$
p_{\mu}=\frac{\partial \Lambda}{\partial \dot{x}^{\mu}}=\frac{\partial \Lambda}{\partial u^{\mu}}=-\frac{m c}{\sqrt{g_{\alpha \beta} \frac{\mathrm{d} x^{\alpha}}{\mathrm{d} \theta} \frac{\mathrm{d} x^{\beta}}{\mathrm{d} \theta}}} g_{\mu \nu} \frac{\mathrm{d} x^{\nu}}{\mathrm{d} \theta} .
$$

Obviously,

$$
p_{\mu}(a u)=p_{\mu}(u)
$$


and this is independent on the parametrization as well. One obtains in the 8dimensional manifold labelled by $\left(x^{\mu}, p_{\mu}\right)$ the 7-dimensional constraints, "energy surface" in the language by Synge, given by the equations

$$
\Omega=g^{\mu \nu} p_{\mu} p_{\nu}-m^{2} c^{2}=0 .
$$

The corresponding Lorentz transformation rules for the four-velocities and fourmomenta have the following form

$$
{ }^{\prime} u^{\mu}=L^{\mu}{ }_{\nu} u^{\nu}, \quad{ }^{\prime} p_{\mu}=p_{\nu} L^{-1 \nu}{ }_{\mu} .
$$

Here $L$ is a Lorentz-homogeneous transformation.

When the electromagnetic field is present, the relativistic Lagrangian is given by

$$
\Lambda=-m c \sqrt{g_{\mu \nu} \frac{\mathrm{d} x^{\mu}}{\mathrm{d} \theta} \frac{\mathrm{d} x^{\nu}}{\mathrm{d} \theta}}+Q A_{\mu} \frac{\mathrm{d} x^{\mu}}{\mathrm{d} \theta} .
$$

Here again $\theta$ is an arbitrary parameter and $g_{\mu \nu}$ is the Minkowskian, or generallyrelativistic, metric tensor of the space-time. Let us remind that in specially-relativistic theory the space-time is a pseudo-Euclidean space $(X, V, \rightarrow ; g)$. Just like in the non-relativistic physics $(X, V, \rightarrow)$ is a four-dimensional affine space and $g \in V^{*} \otimes V^{*}$ is the symmetric pseudo-Euclidean metric tensor of the signature $(+---) . Q$ is the coupling constant, i.e., electric charge in appropriate units. The homogeneous velocity is given by

$$
u^{\mu}=\frac{\mathrm{d} x^{\mu}}{\mathrm{d} \theta}
$$

and the interval and usual velocities are respectively

$$
w^{\mu}=\frac{\mathrm{d} x^{\mu}}{\mathrm{d} s}, \quad v^{\mu}=\frac{\mathrm{d} x^{\mu}}{\mathrm{d} \tau}=\frac{w^{\mu}}{c} .
$$

Here the arc element is expressed as follows

$$
\mathrm{d} s^{2}=g_{\mu \nu} \mathrm{d} x^{\mu} \mathrm{d} x^{\nu}=c^{2} \mathrm{~d} \tau^{2} .
$$

Obviously, Lagrangian (69) is homogeneous of degree one and the corresponding Legendre transformation is four dimensions

$$
p_{\mu}=\frac{\partial \Lambda}{\partial u^{\mu}}
$$

is homogeneous of degree zero

$$
\Lambda(a u)=a \Lambda(u), \quad p_{\mu}(a u)=p_{\mu}(u), \quad a>0 .
$$


The resulting phase-space constraints, i.e., energy equation is given by

$$
\Omega=g^{\mu \nu}\left(p_{\mu}-Q A_{\mu}\right)\left(p_{\nu}-Q A_{\nu}\right)-m^{2} c^{2}=0 .
$$

The four-velocity in any version of the equations (70) or (71) transforms under Lorentz transformations of $(V, g)$ just as it should

$$
{ }^{\prime} u^{\mu}=L^{\mu}{ }_{\nu} u^{\nu}, \quad{ }^{\prime} x_{\mu}=L^{\mu}{ }_{\nu} x^{\nu} .
$$

And the conjugate four-momentum $p_{\mu}$ in(73) transforms under the contragradient rule

$$
{ }^{\prime} p_{\mu}=p_{\nu} L_{\mu}^{-1 \nu}, \quad{ }^{\prime} p_{\mu}{ }^{\prime} u^{\mu}=p_{\mu} u^{\mu} .
$$

\section{Non-Relativistic, Galilean Four-Vector of Momentum}

What concerns Galilean three-velocities and three-momenta, they transform just so under spatial isometries. The problem appears in four dimensions on the level of boosts.

First of all, let us note that according to the formulae (52), (53), (54) energy equals the minus canonical momentum conjugate to the time variable in homogeneous formalism

$$
E=-p_{t}
$$

It is interesting that up to the $c$-multiplier the same is true relativistically, i.e., in the sense of (69), (75)

$$
p_{0}=-\frac{E}{c}=-\frac{m c}{\sqrt{1-v^{2} / c^{2}}}, \quad v^{2}=\delta_{i j} v^{i} v^{j}=-g_{i j} v^{i} v^{j} .
$$

Indeed, we have the rules

$$
\begin{aligned}
& p_{0} x^{0}=p_{0} c t=-E t=-\frac{m c^{2}}{\sqrt{1-v^{2} / c^{2}}} \\
& p_{\mu} x^{\mu}=-E t+\underline{p} \cdot \bar{x}, \quad p_{\mu} \mathrm{d} x^{\mu}=-E \mathrm{~d} t+\underline{p} \cdot \mathrm{d} \bar{x} .
\end{aligned}
$$

As mentioned, the Galilean four-velocity is correctly transformed by the Galilei boosts

$$
\left[\begin{array}{c}
t \\
\bar{x}
\end{array}\right] \mapsto\left[\begin{array}{cc}
1 & \overline{\mathcal{O}}^{T} \\
\bar{\nu} & I_{3}
\end{array}\right]\left[\begin{array}{l}
t \\
\bar{x}
\end{array}\right], \quad\left[\begin{array}{l}
1 \\
\bar{v}
\end{array}\right] \mapsto\left[\begin{array}{cc}
1 & \overline{\mathcal{O}}^{T} \\
\bar{\nu} & I_{3}
\end{array}\right]\left[\begin{array}{l}
1 \\
\bar{v}
\end{array}\right] .
$$

The corresponding dual covector rule reads

$$
\left[\pi ; p_{1}, p_{2}, p_{3}\right] \mapsto\left[\pi ; p_{1}, p_{2}, p_{3}\right]\left[\begin{array}{cc}
1 & \overline{\mathcal{O}}^{T} \\
-\bar{\nu} & I_{3}
\end{array}\right]=\left[\pi-\nu^{i} p_{i} ; p_{1}, p_{2}, p_{3}\right]
$$


It is written for $n=4$, but obviously, the analogous rule holds for the general $n$. But this rule is evidently false! It would mean that the boost transforms $[-E, p]$ into

$$
\left[-^{\prime} E,^{\prime} \underline{p}\right]=[-(E+\underline{p} \cdot \bar{\nu}), \underline{p}]=\left[-\left(\frac{\underline{p}^{2}}{2 m}+\underline{p} \cdot \bar{\nu}\right), \underline{p}\right] .
$$

But physics tell us that for any pair of reference frames

$$
E=\frac{m \bar{v}^{2}}{2}=\frac{\underline{p}^{2}}{2 m}, \quad \underline{p}=m \underline{v}, \quad E^{\prime}=\frac{m \bar{v}^{\prime 2}}{2}=\frac{\underline{p}^{\prime 2}}{2 m}, \quad \underline{p}^{\prime}=m \underline{v}^{\prime}
$$

and the $\bar{\nu}$-boost transition acts as follows

$$
\begin{aligned}
& \bar{v}^{\prime}=\bar{v}+\bar{\nu}, \quad \underline{p}^{\prime}=\underline{p}+m \underline{\nu}=\underline{p}+\underline{\pi} \\
& E^{\prime}=\frac{m(\bar{v}+\bar{\nu})^{2}}{2}=\frac{m \bar{v}^{2}}{2}+m \bar{v} \cdot \bar{\nu}+\frac{m \bar{\nu}^{2}}{2} .
\end{aligned}
$$

Finally we have the following affine transformation rule for the energy-momentum four-covector

$$
\left[-E^{\prime}, \underline{p}^{\prime}\right]=\left[-^{\prime} E,{ }^{\prime} p\right]+\left[-\frac{m \bar{\nu}^{2}}{2}, m \underline{\nu}\right]=\left[-^{\prime} E,{ }^{\prime} p\right]+\left[-\frac{\underline{\pi}^{2}}{2 m}, \underline{\pi}\right] .
$$

Let us remind, that here $\left[-^{\prime} E,^{\prime} \underline{p}\right]$ is the linear-covector part

$$
\left[{ }^{\prime} E,{ }^{\prime} p\right]=[-(E+\underline{p} \cdot \bar{\nu}), \underline{p}]=\left[-\left(E+\underline{p} \cdot \frac{\bar{\pi}}{m}\right), \underline{p}\right] .
$$

The second term in (88), i.e., the additive correction, has a very interesting structure. Namely, it is built of the boost velocity (or momentum) just according to the prescription for the velocity (momentum) dependence of the energy-momentum covector. One can suspect that this rule has something to do with the projective geometry of the Galilei space-time.

\section{Relativistic Theory}

Let us now remind certain peculiarities of the Poincare group, just to review differences in its commutation rules in comparison with the Galilei group and to discuss physical consequences of those differences. As said above, the Minkowskian space $(X, V, \rightarrow ; g)$ has the incomparatively simpler structure than that of the Galilei space. Even literally speaking, its homogeneous-Lorentz part is a simple Lie group! 
Let again $x^{\mu}, \mu=0,1,2,3$, be orthonormal Minkowskian coordinates; we put $x^{0}=c t$, where $t$ is a time variable, $c$ is the velocity of light, and $x^{i}, i=1,2,3$, are the usual spatial coordinates. Therefore

$$
\left[g_{\mu \nu}\right]=\operatorname{diag}(1,-1,-1,-1)
$$

and the arc element is given by

$$
\mathrm{d} s^{2}=\left(\mathrm{d} x^{0}\right)^{2}-\left(\mathrm{d} x^{1}\right)^{2}-\left(\mathrm{d} x^{2}\right)^{2}-\left(\mathrm{d} x^{3}\right)^{2}=c^{2} \mathrm{~d} t^{2}-\mathrm{d} x^{2}-\mathrm{d} y^{2}-\mathrm{d} z^{2} .
$$

Let us mention that in usual physical problems the two conventions $\left(+-{ }_{-}\right)$ and $(-+++)$ are equivalent and each of them has some advantages.

Poincare transformations are ones preserving this structure, so they are linear

$$
{ }^{\prime} x^{\mu}=L^{\mu}{ }_{\nu} x^{\nu}+a^{\mu} .
$$

Here $L$ is the Lorentz transformation

$$
g_{\mu \nu}=g_{\alpha \beta} L_{\mu}^{\alpha} L_{\nu}^{\beta}
$$

In the Galilei space-time there is no absolute space, but there is the absolute time. In Poincare geometry nothing but (90), (91) is absolute and there is a mutual mixing of the space and time variables. Let us illustrate this in the two-dimensional Minkowski space. Lorentz transformations are given by

$$
\left[\begin{array}{l}
{ }^{\prime} x^{0} \\
{ }^{\prime} x^{1}
\end{array}\right]=\left[\begin{array}{cc}
\operatorname{ch} \chi & \operatorname{sh} \chi \\
\operatorname{sh} \chi & \operatorname{ch} \chi
\end{array}\right]\left[\begin{array}{l}
x^{0} \\
x^{1}
\end{array}\right]=L[\chi]\left[\begin{array}{l}
x^{0} \\
x^{1}
\end{array}\right]=\exp \left(\chi\left[\begin{array}{ll}
0 & 1 \\
1 & 0
\end{array}\right]\right)\left[\begin{array}{l}
x^{0} \\
x^{1}
\end{array}\right] .
$$

It is clear that

$$
\operatorname{th} \chi=\frac{\nu}{c}, \quad L\left[\chi_{1}\right] L\left[\chi_{2}\right]=L\left[\chi_{1}+\chi_{2}\right] .
$$

So $\chi$ is a canonical coordinate, $\chi \in(-\infty, \infty)$, and the Lorentz boost $\nu$ has the range $(-c, c)$.

One cannot resist the temptation to quote the explicit historical formula

$$
\left[\begin{array}{l}
{ }^{\prime} t \\
{ }^{\prime} x
\end{array}\right]=L(\nu)\left[\begin{array}{l}
t \\
x
\end{array}\right]=\left[\begin{array}{l}
\frac{1}{\sqrt{1-\nu^{2} / c^{2}}} t+\frac{\nu / c^{2}}{\sqrt{1-\nu^{2} / c^{2}}} x \\
\frac{\nu}{\sqrt{1-\nu^{2} / c^{2}}} t+\frac{1}{\sqrt{1-\nu^{2} / c^{2}}} x
\end{array}\right] .
$$

Obviously, in natural coordinates, when $c=1$, the transformation matrix is symmetric. Similarly, it is difficult not to quote here the composition rule for velocities, i.e., the $\nu$-version of (95) is

$$
L\left(\nu_{1}\right) L\left(\nu_{2}\right)=L(\nu), \quad \nu=\frac{\nu_{1}+\nu_{2}}{1+\left(\nu_{1} / c\right)\left(\nu_{2} / c\right)} .
$$


In a fixed reference frame every Lorentz transformation splits into the product of spatial rotation and boost

$$
L=\mathcal{R}(\bar{k}) \Lambda(\bar{\chi})=\left[\begin{array}{cc}
1 & \overline{\mathcal{O}}^{T} \\
\overline{\mathcal{O}} & R(\bar{k})
\end{array}\right] \Lambda(\bar{\chi}) .
$$

Here

$$
\begin{aligned}
R(\bar{k}) \bar{x} & =\cos k \bar{x}+(1-\cos k) \frac{\bar{k}}{k}\left(\frac{\bar{k}}{k} \cdot \bar{x}\right)+\sin k \frac{\bar{k}}{k} \times \bar{x} \\
\Lambda(\bar{\chi})\left[\begin{array}{c}
x^{0} \\
\bar{x}
\end{array}\right] & =\left[\begin{array}{l}
\operatorname{ch} \chi x^{0}+\operatorname{sh} \chi\left(\frac{\bar{\chi}}{\chi} \cdot \bar{x}\right) \\
\operatorname{sh} \chi \frac{\bar{\chi}}{\chi} x^{0}+\bar{x}+(\operatorname{ch} \chi-1) \frac{\bar{\chi}}{\chi}\left(\frac{\bar{\chi}}{\chi} \cdot \bar{x}\right)
\end{array}\right]
\end{aligned}
$$

and

$$
k=|\bar{k}|, \quad \chi=|\bar{\chi}| .
$$

In spite of certain similarities, a big structural difference is seen just here: rotations $\mathcal{R}$ form a subgroup of $\mathrm{SO}(1,3)$, but the boost - do not do! They may be used as canonical labels of the quotient $\mathrm{SO}(1,3) / \mathrm{SO}(3, \mathbb{R})$. This is nicely seen on the level of infinitesimal commutation rules in the Lie algebra. Namely, let us take infinitesimal operators

$$
\begin{array}{lll}
M_{1}=\left[\begin{array}{cccc}
0 & 0 & 0 & 0 \\
0 & 0 & 0 & 0 \\
0 & 0 & 0 & -1 \\
0 & 0 & 1 & 0
\end{array}\right], & M_{2}=\left[\begin{array}{cccc}
0 & 0 & 0 & 0 \\
0 & 0 & 0 & 1 \\
0 & 0 & 0 & 0 \\
0 & -1 & 0 & 0
\end{array}\right] \\
M_{3}=\left[\begin{array}{cccc}
0 & 0 & 0 & 0 \\
0 & 0 & -1 & 0 \\
0 & 1 & 0 & 0 \\
0 & 0 & 0 & 0
\end{array}\right], & N_{1}=\left[\begin{array}{llll}
0 & 1 & 0 & 0 \\
1 & 0 & 0 & 0 \\
0 & 0 & 0 & 0 \\
0 & 0 & 0 & 0
\end{array}\right] \\
N_{2}=\left[\begin{array}{llll}
0 & 0 & 1 & 0 \\
0 & 0 & 0 & 0 \\
1 & 0 & 0 & 0 \\
0 & 0 & 0 & 0
\end{array}\right], & N_{3}=\left[\begin{array}{llll}
0 & 0 & 0 & 1 \\
0 & 0 & 0 & 0 \\
0 & 0 & 0 & 0 \\
1 & 0 & 0 & 0
\end{array}\right]
\end{array}
$$

respectively for rotations and boosts. The difference in comparison with (24) is obvious. In relativistic rules there appear right-upper units. Because of this, boosts do not form a subgroup. The commutation rules of (101)-(103) have the form

$$
\left[M_{a}, M_{b}\right]=\varepsilon_{a b}^{c} M_{c}, \quad\left[M_{a}, N_{b}\right]=\varepsilon_{a b}^{c} N_{c}, \quad\left[N_{a}, N_{b}\right]=-\varepsilon_{a b}^{c} M_{c} .
$$


The total Poincare group may be described infinitesimally by the following system of $5 \times 5$ matrices

$$
\begin{aligned}
& \mathbb{M}_{1}=\left[\begin{array}{ccccc}
0 & 0 & 0 & 0 & 0 \\
0 & 0 & 0 & 0 & 0 \\
0 & 0 & 0 & -1 & 0 \\
0 & 0 & 1 & 0 & 0 \\
0 & 0 & 0 & 0 & 0
\end{array}\right], \quad \mathbb{M}_{2}=\left[\begin{array}{ccccc}
0 & 0 & 0 & 0 & 0 \\
0 & 0 & 0 & 1 & 0 \\
0 & 0 & 0 & 0 & 0 \\
0 & -1 & 0 & 0 & 0 \\
0 & 0 & 0 & 0 & 0
\end{array}\right] \\
& \mathbb{M}_{3}=\left[\begin{array}{ccccc}
0 & 0 & 0 & 0 & 0 \\
0 & 0 & -1 & 0 & 0 \\
0 & 1 & 0 & 0 & 0 \\
0 & 0 & 0 & 0 & 0 \\
0 & 0 & 0 & 0 & 0
\end{array}\right], \quad \mathbb{N}_{1}=\left[\begin{array}{lllll}
0 & 1 & 0 & 0 & 0 \\
1 & 0 & 0 & 0 & 0 \\
0 & 0 & 0 & 0 & 0 \\
0 & 0 & 0 & 0 & 0 \\
0 & 0 & 0 & 0 & 0
\end{array}\right] \\
& \mathbb{N}_{2}=\left[\begin{array}{lllll}
0 & 0 & 1 & 0 & 0 \\
0 & 0 & 0 & 0 & 0 \\
1 & 0 & 0 & 0 & 0 \\
0 & 0 & 0 & 0 & 0 \\
0 & 0 & 0 & 0 & 0
\end{array}\right], \quad \mathbb{N}_{3}=\left[\begin{array}{lllll}
0 & 0 & 0 & 1 & 0 \\
0 & 0 & 0 & 0 & 0 \\
0 & 0 & 0 & 0 & 0 \\
1 & 0 & 0 & 0 & 0 \\
0 & 0 & 0 & 0 & 0
\end{array}\right] \\
& \mathbb{P}_{1}=\left[\begin{array}{lllll}
0 & 0 & 0 & 0 & 0 \\
0 & 0 & 0 & 0 & 1 \\
0 & 0 & 0 & 0 & 0 \\
0 & 0 & 0 & 0 & 0 \\
0 & 0 & 0 & 0 & 0
\end{array}\right], \quad \mathbb{P}_{2}=\left[\begin{array}{lllll}
0 & 0 & 0 & 0 & 0 \\
0 & 0 & 0 & 0 & 0 \\
0 & 0 & 0 & 0 & 1 \\
0 & 0 & 0 & 0 & 0 \\
0 & 0 & 0 & 0 & 0
\end{array}\right] \\
& \mathbb{P}_{3}=\left[\begin{array}{lllll}
0 & 0 & 0 & 0 & 0 \\
0 & 0 & 0 & 0 & 0 \\
0 & 0 & 0 & 0 & 0 \\
0 & 0 & 0 & 0 & 1 \\
0 & 0 & 0 & 0 & 0
\end{array}\right], \quad \mathbb{P}_{0}=\left[\begin{array}{lllll}
0 & 0 & 0 & 0 & 1 \\
0 & 0 & 0 & 0 & 0 \\
0 & 0 & 0 & 0 & 0 \\
0 & 0 & 0 & 0 & 0 \\
0 & 0 & 0 & 0 & 0
\end{array}\right]=\mathbb{T} \text {. }
\end{aligned}
$$

Their commutation rules have the form $[1,2]$

$$
\begin{aligned}
& {\left[\mathbb{M}_{a}, \mathbb{M}_{b}\right]=\varepsilon_{a b}{ }^{c} \mathbb{M}_{c}, \quad\left[\mathbb{M}_{a}, \mathbb{N}_{b}\right]=\varepsilon_{a b}{ }^{c} \mathbb{N}_{c}, \quad\left[\mathbb{M}_{a}, \mathbb{P}_{b}\right]=\varepsilon_{a b}{ }^{c} \mathbb{P}_{c}} \\
& {\left[\mathbb{N}_{a}, \mathbb{N}_{b}\right]=-\varepsilon_{a b}{ }^{c} \mathbb{M}_{c}, \quad\left[\mathbb{N}_{a}, \mathbb{P}_{b}\right]=\delta_{a b} \mathbb{T}, \quad\left[\mathbb{P}_{a}, \mathbb{P}_{b}\right]=0} \\
& {\left[\mathbb{M}_{a}, \mathbb{T}\right]=0, \quad\left[\mathbb{N}_{a}, \mathbb{T}\right]=\mathbb{P}_{a}, \quad\left[\mathbb{P}_{a}, \mathbb{T}\right]=0 .}
\end{aligned}
$$

It is seen that the difference between the equations (32)-(34) and (110)-(112), is apparently small, is crucial for the drastic distinction between Galilei and relativistic Poincare models. 
Let us now review the drastic difference between Poincare and Galilei classical and quantum physics. First, we repeat the basic formulae

$$
\begin{aligned}
& p_{0}=-\frac{E}{c}=-\frac{m c}{\sqrt{1-v^{2} / c^{2}}}, \quad p_{i}=\frac{m v^{i}}{\sqrt{1-v^{2} / c^{2}}} \\
& p_{\mu} \mathrm{d} x^{\mu}=-E \mathrm{~d} t+\underline{p} \cdot \mathrm{d} \bar{x}=p_{0} c \mathrm{~d} t+\underline{p} \cdot \mathrm{d} \bar{x} .
\end{aligned}
$$

The Hamilton-Jacobi equation, obtained by substituting $\partial S / \partial x^{\mu}$ instead of $p_{\mu}$ in the energy equation, has the following form

$$
g^{\mu \nu}\left(\frac{\partial S}{\partial x^{\mu}}-e A_{\mu}\right)\left(\frac{\partial S}{\partial x^{\nu}}-e A_{\nu}\right)-m^{2} c^{2}=0 .
$$

It is invariant under the second-kind gauge transformations

$$
S \mapsto S+e f, \quad A_{\mu} \mapsto A_{\mu}+\frac{\partial f}{\partial x^{\mu}} .
$$

Without electromagnetism, when only first-kind gauge transformations are admitted, we obtain

$$
g^{\mu \nu} \frac{\partial S}{\partial x^{\mu}} \frac{\partial S}{\partial x^{\nu}}-m^{2} c^{2}=0, \quad f \mapsto f+\text { const. }
$$

This leads to the "heuristic quantization", from the eikonal to the wave equation

$$
p_{\mu} \mapsto \widehat{p}_{\mu}=\mathrm{i} \hbar \frac{\partial}{\partial x^{\mu}}, \quad E \mapsto \mathrm{i} \hbar \frac{\partial}{\partial t}, \quad \underline{p} \mapsto-\mathrm{i} \hbar \bar{\nabla} .
$$

The resulting wave equation is just the Klein-Gordon equation

$$
g^{\mu \nu} \widehat{p}_{\mu} \widehat{p}_{\nu} \Psi=-\hbar^{2} g^{\mu \nu} \partial_{\mu} \partial_{\nu} \Psi=m^{2} c^{2} \Psi .
$$

This is the Casimir eigenequation for the field situations with the fixed mass value. When the electromagnetic field is present, the standard procedure consists in replacing

$$
\partial_{\mu} \mapsto D_{\mu}=\partial_{\mu}+\mathrm{i} e A_{\mu} .
$$

This is compatible with the second-kind gauge invariance

$$
\Psi \mapsto \exp (\mathrm{ief}) \Psi, \quad A_{\mu} \mapsto A_{\mu}+\frac{\partial f}{\partial x^{\mu}} .
$$

Let us now try to express (119) in terms of the "( $3+1)$-apparently non-relativistic" way of writing

$$
-\hbar^{2} \frac{\partial^{2} \Psi}{\partial t^{2}}=\left(-\hbar^{2} c^{2} \bar{\nabla}^{2}+m^{2} c^{4}\right) \Psi=0 .
$$


It turns out, and to be more precise, it follows from the Noether theorem, that the following "continuity equation" holds

$$
\partial_{t} \varrho+\bar{\nabla} \cdot \bar{j}=0
$$

Here

$$
\varrho=\frac{\mathrm{i} \hbar}{2 m c}\left(\bar{\Psi} \partial_{0} \Psi-\left(\partial_{0} \bar{\Psi}\right) \Psi\right), \quad \bar{j}=\frac{\hbar}{2 \mathrm{i} m}(\overline{\Psi \nabla} \Psi-(\overline{\nabla \Psi}) \Psi) .
$$

The probabilistic interpretation fails here, because $\varrho$ is not positively definite. Schrödinger who invented this equation rejected it, but Pauli and Weisskopf accepted it in field the theory where it was derived from the Lagrangian

$$
\mathcal{L}=g^{\mu \nu} \frac{\partial \bar{\Psi}}{\partial x^{\mu}} \frac{\partial \Psi}{\partial x^{\nu}} \sqrt{|g|}-\frac{m^{2} c^{2}}{\hbar^{2}} \bar{\Psi} \Psi \sqrt{|g|} .
$$

It is invariant under the first kind gauge symmetry $\Psi \mapsto \exp (\mathrm{i} \chi) \Psi$. Localizing it, i.e., taking $\chi$ to be a function of the space-time point, we include interaction with the electromagnetic field and replace $(125)$ by

$$
\mathcal{L}=g^{\mu \nu} D_{\mu} \bar{\Psi} D_{\nu} \Psi \sqrt{|g|}-\frac{m^{2} c^{2}}{\hbar^{2}} \bar{\Psi} \Psi \sqrt{|g|} .
$$

Here the covariant derivative $D_{\mu}$ is given by (120). The Noether four-current for (125) is given by (124), i.e., in the consequent four-dimensional language by

$$
j^{\mu}=\mathrm{ieg} g^{\mu \nu}\left(\bar{\Psi} \partial_{\nu} \Psi-\left(\partial_{\nu} \bar{\Psi}\right) \Psi\right) \sqrt{|g|}
$$

or in the gauge-invariant version by

$$
j^{\mu}=\operatorname{ieg}^{\mu \nu}\left(\bar{\Psi} D_{\nu} \Psi-\left(D_{\nu} \bar{\Psi}\right) \Psi\right) \sqrt{|g|} .
$$

This leads to the charge functional

$$
(\Psi, \Sigma) \mapsto Q[\Psi, \Sigma]
$$

Here $\Sigma$ is a spatial surface extended to spatial infinity, e.g., $t=$ const and $Q[\Psi, \Sigma]$ is given by the integral over $\Sigma$

$$
Q[\Psi, \Sigma]=\int j^{\mu}(\Psi, \partial \Psi) \mathrm{d} \sigma_{\mu}
$$

On the basis of the continuity equation

$$
\partial_{\mu} j^{\mu}=0
$$


following from the field equations, $Q$ does not depend on $\Sigma$ (on time). This is the charge conservation

$$
\frac{\partial Q[\Psi]}{\partial t}=0
$$

The expression $Q[\Psi]$ is a quadratic form of the field $\Psi$. One can perform its polarization procedure which leads from the quadratic form $u \mapsto Q[u]$ to the sesquilinear one $(u, v) \mapsto \breve{Q}[u, v]$ according to the rule

$$
4 \breve{Q}[u, v]=Q[u+v]-Q[u-v]-\mathrm{i} Q[u+\mathrm{i} v]+\mathrm{i} Q[u-\mathrm{i} v] .
$$

The resulting expression is just the Klein-Gordon scalar product of fields

$$
\begin{aligned}
(\Psi, \varphi) & =\mathrm{i} \int_{\Sigma} \mathrm{d} \sigma^{\mu}(x) \bar{\Psi}(x) \overleftrightarrow{\partial_{\mu}} \varphi(x) \\
& =\mathrm{i} \int_{\Sigma} \mathrm{d} \sigma^{\mu}(x)\left(\bar{\Psi}(x) \partial_{\mu} \varphi(x)-\left(\partial_{\mu} \bar{\Psi}\right)(x) \varphi(x)\right)
\end{aligned}
$$

which does not depend on $\Sigma$ (on time). Using the positive-frequency expression

$$
\Psi(x)=\frac{\sqrt{2}}{(2 \pi)^{3 / 2}} \int \mathrm{d}^{4} k \exp \left(-\mathrm{i} k_{\mu} x^{\mu}\right) \delta\left(k^{2}-m^{2}\right) \theta\left(k_{0}\right) \chi(k)
$$

where $\theta$ is the Heaviside function, i.e.,

$$
\begin{aligned}
& \Psi(x)=\int \mathrm{d} \mu_{m}^{\uparrow}(\bar{k}) \chi(\bar{k}) \exp (-\mathrm{i}(\omega(\bar{k}) t-\bar{k} \cdot \bar{x})) \\
& \omega(\bar{k})=k_{0}=\sqrt{\bar{k}^{2}+m^{2}}, \quad \mathrm{~d} \mu_{m}(\bar{k})=\frac{\mathrm{d}^{3} \bar{k}}{\omega_{m}(\bar{k})}
\end{aligned}
$$

we express (135) as

$$
\left(\Psi_{1} \mid \Psi_{2}\right)=\int \mathrm{d} \mu_{m}^{\uparrow}(\bar{k}) \bar{\chi}_{1}(\bar{k}) \chi_{2}(\bar{k}) .
$$

The operation $U(\Lambda, a)$ given by

$$
(U(\Lambda, a) \Psi)(x)=\Psi\left(\Lambda^{-1}(x-a)\right)
$$

is unitary with respect to (135), (138)

$$
\left(U(\Lambda, a) \Psi_{1} \mid U(\Lambda, a) \Psi_{2}\right)=\left(\Psi_{1} \mid \Psi_{2}\right)
$$


if $\Psi_{1}, \Psi_{2}$ satisfy the same field equation. The representation space is characterized by the same fixed value $m^{2}$ of the Casimir invariant $g^{\mu \nu} P_{\mu} P_{\nu}$. Obviously, the operation (139) may be easily expressed in terms of the field profile $\chi(\bar{k})$ defined on the mass shell $g_{\mu \nu} k^{\mu} k^{\nu}=m^{2}$ (in the natural units, when we put $\hbar=1$ )

$$
(U(\Lambda, a) \Psi)(x)=\int \mathrm{d} \mu_{m}(k)(U\{\Lambda, a\} \chi)(k) \exp \left(-\mathrm{i} k_{\mu} x^{\mu}\right)
$$

namely

$$
(U\{\Lambda, a\} \chi)(k)=\exp \left(\mathrm{i} a^{\mu} k_{\mu}\right) \chi\left(k_{\mu} \Lambda_{\mu}^{-1 \nu}\right)
$$

\section{Non-Relativistic Strange Features, Projective Representation}

Let us now go back to the Galilei group framework, more precisely to the free, i.e., potential-independent, Schrödinger equation

$$
\mathrm{i} \hbar \frac{\partial \Psi}{\partial t}+\frac{\hbar^{2}}{2 m} \triangle \Psi=0
$$

Obviously, its solution may be Fourier-represented, just like (135)

$$
\Psi(t, \bar{x})=\int \frac{\mathrm{d}^{3} \underline{p}}{(2 \pi \hbar)^{3}} \varphi(\underline{p}) \exp \left(-\frac{\mathrm{i}}{\hbar}(E t-\underline{p} \cdot \bar{x})\right) .
$$

But obviously, the Galilei transformation cannot act on them according to the pointwise rule like in the relativistic Klein-Gordon and other equations

$$
(U[\mathcal{G}] \Psi)(t, \bar{x}) \neq \Psi\left(\mathcal{G}^{-1}(t, \bar{x})\right)
$$

just because of the covector failure of boosts.

But the Fourier representation, slightly similar to (141), (142), although different in important details, may be used. Indeed, taking the true physical transformation of the energy-momentum, we can transform the wave amplitudes of definite energymomentum values as follows

$$
\Psi_{(E, \underline{p})}=\exp \left(-\frac{\mathrm{i}}{\hbar}(E t-\underline{p} \cdot \bar{x})\right) \mapsto \Psi_{\left(E^{\prime}, \underline{p}^{\prime}\right)}=\exp \left(-\frac{\mathrm{i}}{\hbar}\left(E^{\prime} t-\underline{p}^{\prime} \cdot \bar{x}\right)\right) .
$$

Here

$$
E=\frac{\underline{p}^{2}}{2 m}, \quad E \prime=\frac{\underline{p}^{\prime 2}}{2 m}
$$


Therefore, on the eigenfuncitons (146) the boost mappings act as follows: $\Psi \mapsto \Psi^{\prime}$, where

$$
\Psi^{\prime}(t, \bar{x})=\exp \left(-\frac{\mathrm{i}}{\hbar} m\left(\frac{\bar{\nu}^{2}}{2} t-\bar{\nu} \cdot \bar{x}\right)\right) \Psi(t, \bar{x}-\bar{\nu} t) .
$$

And for the general space-time wave functions we have

$$
(U\{\bar{\nu}\} \Psi)(t, \bar{x})=\exp \left(-\frac{\mathrm{i}}{\hbar} m\left(\frac{\bar{\nu}^{2}}{2} t-\bar{\nu} \cdot \bar{x}\right)\right) \Psi(t, \bar{x}-\bar{\nu} t) .
$$

Therefore, the proper action involves both the action on the space-time argument, but also the multiplication of the wave function by some local, $(t, \bar{x})$-dependent term. The same is true for the general wave functions, not only those satisfying the potential-free Schrödinger equation. The other, non-boost transformations act in the usual argument-wise actions.

When dealing on free evolutions, we can use the pointwise action on the wave profiles. Namely, without the $t$-variable

$$
\Psi(\bar{x})=\frac{1}{(2 \pi \hbar)^{3}} \int \mathrm{d}^{3} \underline{p} \varphi(\underline{p}) \exp \left(\frac{\mathrm{i}}{\hbar} \underline{p} \cdot \bar{x}\right)
$$

we define the action of the Newton translations as follows

$$
U[\bar{\alpha}] \Psi(\bar{x})=\Psi(\bar{x}-\bar{\alpha}) .
$$

So, the usual argument action of the spatial translations. However, the boost operations act pointwisely on the wave profiles

$$
(V[\bar{\nu}] \Psi)(\bar{x})=\frac{1}{(2 \pi \hbar)^{3}} \int \mathrm{d}^{3} \underline{p}(V\{\bar{\nu}\} \varphi)(\underline{p}) \exp \left(\frac{\mathrm{i}}{\hbar} \underline{p} \cdot \bar{x}\right)
$$

and

$$
(V\{\bar{\nu}\} \varphi)(\underline{p})=\varphi(\underline{p}-m \bar{\nu}) .
$$

Therefore,

$$
(V[\bar{\nu}] \Psi)(\bar{x})=\exp \left(\frac{\mathrm{i}}{\hbar} m \bar{\nu} \cdot \bar{x}\right) \Psi(\bar{x})=\exp \left(\frac{\mathrm{i}}{\hbar} \bar{\pi} \cdot \bar{x}\right) \Psi(\bar{x}) .
$$

The Weyl operators of the Newton translations act as

$$
\mathcal{W}[\bar{\alpha}, \bar{\nu}]=U[\bar{\alpha}] V[\bar{\nu}] .
$$


However it is more convenient to use the modified Weyl operators

$$
W[\bar{\alpha}, \bar{\nu}]=\exp \left(\frac{\mathrm{i}}{2 \hbar} m \bar{\nu} \cdot \bar{\alpha}\right) \mathcal{W}[\bar{\alpha}, \bar{\nu}] .
$$

Their advantage is the nice expression for inverses

$$
W[\alpha, \bar{\nu}]=W[-\bar{\alpha},-\bar{\nu}]
$$

and the nice symplectic composition form

$$
W\left[\bar{\alpha}_{1}, \bar{\nu}_{1}\right] W\left[\bar{\alpha}_{2}, \bar{\nu}_{2}\right]=\exp \left(\frac{\mathrm{i} m}{2 \hbar}\left(\bar{\nu}_{1} \cdot \bar{\alpha}_{2}-\bar{\nu}_{2} \cdot \bar{\alpha}_{1}\right) W\left[\bar{\alpha}_{1}+\bar{\alpha}_{2}, \bar{\nu}_{1}+\bar{\nu}_{2}\right]\right.
$$

i.e.,

$$
W\left[\bar{u}_{1}\right] W\left[\bar{u}_{2}\right]=\exp \left(\frac{\mathrm{i}}{2 \hbar} m \Gamma_{g}\left(\bar{u}_{1}, \bar{u}_{2}\right)\right) W\left[\bar{u}_{1}+\bar{u}_{2}\right] .
$$

Here the matrix of $\Gamma_{g}$ is the usual symplectic two-form

$$
\Gamma_{g}=\left[\begin{array}{cc}
\mathcal{O}_{n} & -I_{n} \\
I_{n} & \mathcal{O}_{n}
\end{array}\right]
$$

This is the projective representation of the Newton group, in canonical Bargmann form based on the skew-symmetric matrices in the factor [3,6-10]. Let us stress a very important point which witnesses about the essential discontinuity of the non-relativistic limit. In relativistic theory the square of mass is the value of the projector operator. But in non-relativistic theory it is something completely else. Namely, it is a parameter which labels the projective factors of ray representation.

Let us quote some infinitesimal rules. So, we introduce generators of translations and boosts

$$
\begin{aligned}
& U[\bar{\alpha}] \underset{\alpha \underset{\approx}{\approx} 0}{\approx} \operatorname{Id}-\alpha^{k} \frac{\partial}{\partial x^{k}}=\operatorname{Id}-\alpha^{k} \frac{\mathrm{i}}{\hbar} P_{k}, \quad P_{k}=\frac{\hbar}{\mathrm{i}} \frac{\partial}{\partial x^{k}} \\
& V[\bar{\nu}] \underset{\bar{\nu} \approx 0}{\approx} \operatorname{Id}+\nu^{k} \frac{\mathrm{i}}{\hbar} m x^{k}=\mathrm{Id}+\nu^{k} \frac{\mathrm{i}}{\hbar} B_{k}=I+\nu^{k} \frac{\mathrm{i}}{\hbar} m Q_{k} .
\end{aligned}
$$

Obviously, the generators are simply operators of momentum and position, and

$$
\frac{1}{\mathrm{i} \hbar}\left[Q^{a}, P_{b}\right]=\delta^{a}{ }_{b}
$$

Let us introduce the corresponding adjoint transformations

$$
\begin{aligned}
& \operatorname{Ad}_{U[\bar{\alpha}]} \varrho=U[\bar{\alpha}] \varrho U[\bar{\alpha}]^{-1}, \quad \operatorname{ad}_{P_{k}} \varrho=\frac{1}{\mathrm{i} \hbar}\left[P_{k}, \varrho\right] \\
& \operatorname{Ad}_{V[\bar{\nu}]} \varrho=V[\bar{\nu}] \varrho V[\bar{\nu}]^{-1}, \quad \operatorname{ad}_{Q^{k}} \varrho=\frac{1}{\mathrm{i} \hbar}\left[Q^{k}, \varrho\right] .
\end{aligned}
$$


Let us notice that those adjoint operators mutually commute

$$
\operatorname{Ad}_{U[\bar{\alpha}]} \operatorname{Ad}_{V[\bar{\nu}]}=\operatorname{Ad}_{V[\bar{\nu}]} \operatorname{Ad}_{U[\bar{\alpha}]}, \quad\left[\operatorname{ad}_{Q^{k}}, \operatorname{ad}_{P_{l}}\right]=0 .
$$

The non-commutativity of position and momentum has geometric reasons: it is impossible with $m$ degrees of freedom to have more than the $m$-members systems of functionally independent generators. This is the reason that the Galilei group for massive particles admits only projective unitary representations. This peculiarity underlies the Weyl-Wigner-Moyal-Ville phase space formulation of quantum mechanics.

Namely, let us take a kind of the group-algebraic linear shells of $(156)[4,5,13,14]$

$$
\begin{aligned}
\mathbb{A} & =\int \widehat{A}(\bar{\alpha}, \bar{\pi}) W[\bar{\alpha}, \bar{\pi}] \mathrm{d}^{3} \bar{\alpha} \frac{\mathrm{d}^{3} \bar{\pi}}{(2 \pi \hbar)^{3}} \\
& =\int \widehat{A}(\bar{\alpha}, \bar{\pi}) \exp \left(\frac{\mathrm{i}}{\hbar}\left(\pi_{a} Q^{a}+\alpha^{a} P_{a}\right)\right) \mathrm{d}^{3} \bar{\alpha} \frac{\mathrm{d}^{3} \bar{\pi}}{(2 \pi \hbar)^{3}} .
\end{aligned}
$$

Here $\bar{\pi}=m \bar{\nu}$ and $\widehat{A}$ is the Fourier transform of a classical phase space function $A$,

$$
A(\bar{x}, \underline{p})=\int \widehat{A}(\bar{\alpha}, \bar{\pi}) \exp \left(\frac{\mathrm{i}}{\hbar}\left(\pi_{a} x^{a}+\alpha^{a} p_{a}\right)\right) \mathrm{d}^{3} \bar{\alpha} \frac{\mathrm{d}^{3} \bar{\pi}}{(2 \pi \hbar)^{3}} .
$$

Obviously, the value $m=3$ of degrees of freedom is here quite accidental, it may be any $m$.

The operators $\mathbb{A}, \mathbb{B}$ represent the classical functions $A, B$. Similarly

$$
\{\mathbb{A}, \mathbb{B}\}_{\mathrm{QPB}}=\frac{1}{\mathrm{i} \hbar}[\mathbb{A}, \mathbb{B}]
$$

are in terms of the phase space functions represented by

$$
A *_{\text {Weyl }} B, \quad\{A, B\}_{\text {Weyl-Moyal }} .
$$

Let us quote a few explicit expressions, like the group commutators

$$
\begin{aligned}
W\left[\bar{u}_{1}\right] W\left[\bar{u}_{2}\right] W\left[\bar{u}_{1}\right]^{-1} W\left[\bar{u}_{2}\right]^{-1} & =\exp \left(\frac{\mathrm{i}}{\hbar} m \Gamma_{g}\left(\bar{u}_{1}, \bar{u}_{2}\right)\right) \operatorname{Id} \\
W\left\{\bar{z}_{1}\right\} W\left\{\bar{z}_{2}\right\} W\left\{\bar{z}_{1}\right\}^{-1} W\left\{\bar{z}_{2}\right\}^{-1} & =\exp \left(\frac{\mathrm{i}}{\hbar} m \Gamma\left(\bar{z}_{1}, \bar{z}_{2}\right)\right) \mathrm{Id} .
\end{aligned}
$$

The first expression, based on the $(\bar{\alpha}, \bar{\nu})$ representation is in a sense more natural than that based on $(\bar{\alpha}, \underline{\pi})$. 
The Weyl product may be expressed as follows

$$
(A * B)(\bar{z})=2^{2 n} \int \mathrm{e}^{\frac{2 \mathrm{i} m}{\hbar} \Gamma\left(\bar{z}-\bar{z}_{1}, \bar{z}-\bar{z}_{2}\right)} A\left(\bar{z}_{1}\right) B\left(\bar{z}_{2}\right) \mathrm{d} \mu\left(\bar{z}_{1}\right) \mathrm{d} \mu\left(\bar{z}_{2}\right) .
$$

Here

$$
\mathrm{d} \mu(\bar{z})=\mathrm{d} \mu(\bar{q}, \underline{p})=\frac{1}{(2 \pi \hbar)^{n}} \mathrm{~d} q^{1} \ldots \mathrm{d} q^{m} \mathrm{~d} p_{1} \ldots \mathrm{d} p_{m} .
$$

The relationship between phase-space functions and operators may be expressed in a suggestive way by using the kernels of operators

$$
A\left[q, q^{\prime}\right]=\left\langle q|\mathbb{A}| q^{\prime}\right\rangle, \quad(\mathbb{A} \Psi)(q)=\int A\left[q, q^{\prime}\right] \Psi\left(q^{\prime}\right) \mathrm{d}^{m} q^{\prime}
$$

Namely, one can show that

$$
A\left[q, q^{\prime}\right]=\int \exp \left(\frac{\mathrm{i}}{\hbar} \underline{p} \cdot\left(\bar{q}-\bar{q}^{\prime}\right)\right) A\left(\frac{1}{2}\left(\bar{q}+\bar{q}^{\prime}\right), \underline{p}\right) \frac{\mathrm{d}^{m} \underline{p}}{(2 \pi \hbar)^{m}}
$$

and conversely

$$
A(q, p)=\int \exp \left(-\frac{\mathrm{i}}{\hbar} \underline{p} \cdot \bar{\alpha}\right) A\left[\bar{q}+\frac{\bar{\alpha}}{2}, \bar{q}-\frac{\bar{\alpha}}{2}\right] \mathrm{d}^{n} \bar{\alpha} .
$$

All those formulas are affinely-invariant. And everything follows from the structure of Galilei group and the boost failure.

\section{Final Remarks}

Let us finish with some related remarks concerning Hamilton generators. Let us denote the Hamiltonian vector fields

$$
X_{F}=\left[\cdots, \frac{\partial F}{\partial p_{i}}, \cdots ; \cdots,-\frac{\partial F}{\partial q^{i}}, \cdots\right] .
$$

Let us consider a Lie algebra of such vectorfields, in the sense of Lie brackets

$$
\left[X_{A}, X_{B}\right]=C_{A B}^{D} X_{D}
$$

Here

$$
[X, Y]^{a}=X^{b} Y_{, b}^{a}-Y^{b} X^{a}{ }_{, b} .
$$

There is a natural question: do exist such functions $F_{A}$ that the following holds

$$
X_{A}=X\left(F_{A}\right), \quad\left\{F_{A}, F_{B}\right\}=C_{A B}^{D} F^{D} .
$$


The answer is no! And this is the case with generators of the Newton group. For semisimple group the answer is always affirmative, but for non-semisimple with all both embarrassing but also interesting consequences. In general instead (181) we have only

$$
\left\{F_{A}, F_{B}\right\}=C_{A B}^{D} F_{D}+\omega_{A B}, \quad \omega_{A B}=-\omega_{B A} .
$$

And in the non-semisimple group it may happen (although need not) that no change of basis may eliminate the coefficients $\omega_{A B}$.

\section{Acknowledgements}

We are very grateful to the Ministry of Science and Higher Education in Poland for the research grant N N501 049540 financed from the Scientific Research Support Fund in 2011-2014 which is very helpful in our work and connections with Bulgarian scientists. The thanks are also due to the Bulgarian and Polish Academies of Sciences for supporting our exchange project. And of course to professor Ivaïlo M. Mladenov for his cordial scientific and moral help and for his understanding.

\section{References}

[1] Abraham R. and Marsden J., Foundations of Mechanics, Benjamin/Cummings, London-Amsterdam-Sydney-Tokyo 1978.

[2] Arnold V., Mathematical Methods of Classical Mechanics, Springer Graduate Texts in Mathematics 60, Springer, New York 1978.

[3] Bargmann V., On Unitary Ray Representations of Continuous Groups, Ann. Math. 59 (1954) 1-46.

[4] Moyal J., Quantum Mechanics as a Statistical Theory, Proc. Camb. Phil. Soc. 45 (1949) 99-124.

[5] Sławianowski J.J., Uncertainty, Correspondence and Quasiclassical Compatibility, In: The Uncertainty Principle and Foundations of Quantum Mechanics. A Fifty Years Survey. A tribute to Professor Werner Heisenberg to commemorate the fiftieth anniversary of the formulation of quantum mechanics, W. Price and S. Schissick (Eds), John Wiley \& Sons, London-New YorkSydney-Toronto 1977, pp 147-188.

[6] Sławianowski J.J., Geometry of Phase Spaces, PWN - Polish Scientific Publishers, Warsaw and John Wiley \& Sons, Chichester 1991. 
[7] Sławianowski J.J., Kovalchuk V., Martens A., Gołubowska B. and Rożko E. E., Quasiclassical and Quantum Systems of Angular Momentum. Part I. Group Algebras as a Framework for Quantum-Mechanical Models with Symmetries, J. Geom. Symmetry Phys. 21 (2011) 61-94.

[8] Sławianowski J.J., Kovalchuk V., Martens A., Gołubowska B. and Rożko E. E., Quasiclassical and Quantum Systems of Angular Momentum. Part II. Quantum Mechanics on Lie Groups and Methods of Group Algebras, J. Geom. Symmetry Phys. 22 (2011) 67-94.

[9] Sławianowski J.J., Kovalchuk V., Martens A., Gołubowska B. and Rożko E. E., Quasiclassical and Quantum Systems of Angular Momentum. Part III. Group Algebra su(2), Quantum Angular Momentum and Quasiclassical Asymptotics, J. Geom. Symmetry Phys. 23 (2011) 59-95.

[10] Sławianowski J.J., Kovalchuk V., Martens A., Gołubowska B., Rożko E. E., Quasiclassical and Quantum Dynamics of Systems of Angular Momenta, In: Geometry, Integrability and Quantization XII, Ivailo M. Mladenov, Gaetano Vilasi and Akira Yoshioka (Eds), Avangard Prima, Sofia 2011, pp 70-155.

[11] Synge J., Geometrical Mechanics and de Broglie Waves, Cambridge Univ. Press, London 1954.

[12] Synge J., Classical Dynamics, In: Handbuch der Physik, S. Flügge (Ed), Springer, Berlin 1960.

[13] Weyl H., Quantenmechanik und Gruppentheorie, Zeitschrift für Physik 46 (1927) 1-46.

[14] Weyl H., The Theory of Groups and Quantum Mechanics, Dover, New York 1950.

Institute of Fundamental

Technological Research

Polish Academy of Sciences

Pawińskiego Str. $5^{\mathrm{B}}$

02-106 Warsaw, POLAND

Barbara Gołubowska - E-mail address: bgolub@ ippt . gov • pl

Vasyl Kovalchuk - E-mail address: vkoval @ippt.gov •pl

Agnieszka Martens - E-mail address: amartens@ippt.gov.pl

Ewa Eliza Rożko - E-mail address: erozko@ippt.gov •pl

Jan Jerzy Sławianowski - E-mail address: js lawian@ippt.gov.pl 